\title{
Les lois spéciales à caractère régional dans le code des Qing
}

On Special Laws with a Regional Character in the Qing Code

論清代條例中的地區性特别法

\section{Zhiqiang Wang}

\section{OpenEdition}

\section{Journals}

Édition électronique

URL : http://journals.openedition.org/extremeorient/608

DOI : $10.4000 /$ extremeorient.608

ISBN : 978-2-84292-558-1

ISSN : 2108-7105

\section{Éditeur}

Presses universitaires de Vincennes

\section{Édition imprimée}

Date de publication : 21 novembre 2016

Pagination : 103-126

ISBN : 978-2-84292-556-7

ISSN : 0754-5010

\section{Référence électronique}

Zhiqiang Wang, "Les lois spéciales à caractère régional dans le code des Qing », Extrême-Orient Extrême-Occident [En ligne], 40 | 2016, mis en ligne le 21 novembre 2018, consulté le 01 mai 2019. URL : http://journals.openedition.org/extremeorient/608; DOI : 10.4000/extremeorient.608 


\title{
Les lois spéciales à caractère régional dans le code des Qing*
}

\author{
Wang Zhiqiang
}

L'ancien droit chinois contient une quantité non négligeable de lois spéciales à caractère régional. Il s'agit de règles promulguées par l'administration centrale au nom de l'empereur pour des relations juridiques spécifiques à certaines régions, et différentes de celles applicables en d'autres lieux. Tout en étant largement présentes dans les codes en vigueur dans le reste du pays, elles sont en réalité applicables uniquement à une région déterminée ce qui montre clairement que ce sont des règles spéciales de nature régionale. Le présent article a pour objet les sources historiques conservant de façon relativement complète les articles additionnels dans le code des Qing (1644-1911) ${ }^{1}$. Il présente des statistiques sur le nombre de lois régionales spéciales, leur

* Texte adapté du chinois par Frédéric Constant et Jérôme Bourgon. La technicité de l'article a rendu nécessaire certains ajouts, ainsi que des notes des traducteurs, indiquées par les caractères NDT. Les cartes de cet article ne figurent pas originellement dans le travail de Wang Zhiqiang. Elles ont été réalisées par Estelle Senna, géomaticienne à l'Institut d'Asie Orientale de l'ENS de Lyon, à partir de données recueillies par Che Qun 车群, actuellement professeur associé au département d'histoire de la Shanghai Jiaotong University.

1. NDT. Les éditions du Code des Grands Qing (Da Qing lüli) propres à chaque règne étaient nombreuses. En leur sein, les articles additionnels (tiaoli) faisaient nominalement l'objet d'une petite révision tous les cinq ans et d'une grande tous les dix, mais des variations existent selon les règnes. Néanmoins, à partir de 1870 jusqu'aux réformes de la fin des Qing, il n'y eut plus de révision systématique. À la fin de l'ère Guangxu (1875-1908), les précédents établis lors des règnes antérieurs ainsi que les règlements éparses atteignirent des quantités impressionnantes, dépassant la production des précédentes dynasties. Le juriste Xue Yunsheng (1820-1901) fit paraître à cette époque les Doutes persistant à la lecture des lois, ouvrage présentant de façon relativement complète les articles alors en vigueur ainsi que l'analyse de leur évolution. En raison de sa très grande valeur, des éditions modernes ont été publiées depuis, la principale étant celle de Huang Jingjia ( $c f$. Xue 1970). Les articles étudiés 
répartition, leur situation, leur origine, leur contenu, ainsi que leur relations étroites avec la centralisation du pouvoir. Il propose enfin une première analyse critique de leur signification.

\section{Cadre et objet de la présente étude}

Il importe de bien saisir la spécificité de notre objet, qui n'est pas, comme on pourrait s'y attendre, un ensemble de lois ou de règlements produits par des organismes locaux en fonction de besoins locaux. Bien au contraire, les « lois spéciales à caractère régional » (diquxing tebie fa) étaient promulguées par l'administration centrale. Elles visaient à réprimer tout acte commis par un « justiciable ordinaire » (yiban zhuti) ${ }^{2}$, sans qu'il s'y attache aucun caractère régional particulier. Simplement, lorsque l'administration centrale estimait que des circonstances particulières à cette région devaient être prises en compte, elle produisait une loi spéciale à cette région. Par «justiciable ordinaire», il faut entendre un type de justiciables qu'on rencontre à l'identique sur l'ensemble du territoire, et non pas seulement dans une certaine région. Les lois spéciales à caractère régional dont il est question dans cet article ne comprennent pas les normes qui ont été instituées pour des justiciables spécifiques à une région donnée. Ainsi, par exemple, un article additionnel inséré en 1758 dans la section «Infractions sexuelles » (fanjian) du Code des Grands Qing dispose que :

dans cet article, leur numérotation, ainsi que les chiffres de nos statistiques, sont basés sur cette édition.

2. NDT. yiban zhuti est un terme bien particulier au droit pénal chinois désignant la personne naturelle (ziran ren) qui est en capacité de commettre des infractions " générales », ou « ordinaires », comme les diverses catégories d'homicides, ou de vols, et qui remplit les conditions nécessaires (âge, santé mentale, etc.) pour en assumer la responsabilité pénale - d'où notre traduction par « justiciable ordinaire ». Une traduction plus littérale, mais moins explicite, serait « sujet de droit ordinaire ». Le terme a un antonyme : teshu zhuti, qui désigne ceux des justiciables (ou « sujets de droit ») qui nécessitent un traitement spécifique (teshu), soit du fait de leur statut (militaires, gestionnaires de biens publics), soit du fait des actes commis (détournement d'armes ou autres biens publics). Dans notre article, les « justiciables spéciaux », comme les Bandits Gu du Sichuan, ou les « actes spécifiques à une région » comme l'estimation des digues du Sichuan, ne sont pas du ressort des « lois spéciales à caractères régional ». 
Dans les affaires de viol en réunion commis par les bandits $\mathrm{Gu}$ au Sichuan [...], même si le viol n'a pas encore été consommé par les co-auteurs, il faut les condamner à la strangulation sous réserve de révision par les Assises d'automne, en vertu de l'article sur le viol en réunion.

Le commentaire de Xue Yunsheng dans son ouvrage Doutes persistants à la lecture des articles additionnels du code des Qing (Duli cunyi, ci-après Commentaires de M. Xue) indique que :

La règle commune pour le viol en réunion, c'est que l'auteur principal est condamné à la décapitation immédiate, et les auteurs secondaires à la strangulation sous réserve de révision par les Assises d'automne. Toutefois, si le viol n'a pas été consommé, les auteurs sont simplement condamnés une peine d'exil. Dans le cas présent, alors que le viol n'a pas été consommé, la peine est la strangulation pour tous les co-auteurs : c'est parce que ce sont des bandits Gu qu'ils sont punis plus sévèrement ${ }^{3}$.

Le terme bandit $\mathrm{Gu}$ désigne une société secrète du Sichuan, les Guluzi (également appelé «bande des Gulu») ${ }^{4}$. Ces actes sont commis non par des « justiciables ordinaires », mais par des justiciables bien spécifiques au Sichuan, ils ne faisaient donc pas l'objet d'une « loi spéciale à caractère régional ».

Par « actes non spécifiques à un territoire », il faut entendre des actes susceptibles de se produire sur n'importe quelle partie du territoire national. Faute de quoi, les règlements correspondants ne peuvent être considérés comme des « lois spéciales à caractère régional ». Ainsi, dans la partie « Lois militaires » du code des Qing, les actes touchant à la violation des temples des ancêtres ou des palais impériaux ne peuvent avoir lieu ailleurs que dans la région de Pékin. De même, un article additionnel ajouté en 1735 à l'article «Consommation frauduleuse des matériaux et objets » dispose que : «Pour les constructions et réfections de digues dans la province du Zhejiang, l'estimation

3. Xue $1970: 1085$ (Art. 366-08).

4. NDT. Ces bandits étaient désignés d'un caractère chinois dont la prononciation est guo en mandarin, mais dont la prononciation locale était $g u$ : d'où «Bandits $\mathrm{Gu}$ » ou « Guluzi ». Il s'agissait d'anciens habitants du Sichuan chassés par les troubles de la fin des Ming et l'invasion Mandchoue. À leur retour, ils trouvèrent leurs terres occupées par les nouveaux maîtres de la Chine. Les syllabes «gu » et «lu » imiteraient le parler guttural des bandits et les intonations plaintives des mendiants qu'ils étaient devenus. Pour les fonctionnaires des Qing, ces marginaux ne représentaient pas une menace suffisamment « générale » ou « ordinaire » pour faire l'objet d'une « loi spéciale à caractère régional ». 
du prix se fait en taels d'argent...» Selon les Commentaires de M.Xue : «Cela désigne spécifiquement les digues marines de la province du Zhejiang ${ }^{5}$. » L'objet, le lieu et le caractère de ces actes ont tous un caractère spécifiquement local, qui n'a pas d'équivalent dans d'autres régions : ils n'entrent donc pas dans notre définition des "lois spéciales à caractère régional». En outre, les territoires désignés par des appellations indirectes et peu claires, comme " province extérieure », « mission extérieure », « arrière-pays », " Chine de l'intérieur », etc., n'entrent pas dans le cadre de cette étude.

Sous les Qing, les articles additionnels qui étaient périodiquement intégrés au code pénal étaient tous promulgués au nom de l'empereur. Parmi eux, un nombre non négligeable était en vigueur dans une région déterminée, et non sur l'ensemble du territoire, ce qui en fait typiquement des « lois spéciales à caractère régional ». Comme ces lois ont été intégrées au code des Qing en tant qu'articles additionnels (tiaoli) aux articles principaux (lii), nous les appellerons aussi «articles additionnels spéciaux à caractère régional ». Ils sont différents de ce que la science du droit moderne appelle « règlements locaux » en ce que ces derniers sont produits par des organes législatifs locaux. Pour nous résumer, le terme bien spécifique de « lois spéciales à caractère régional » s'applique à des mesures prises par l'administration centrale pour traiter un problème ressenti comme « général » et d'importance stratégique, tel qu'il se posait dans un lieu particulier.

Si l'on se fonde sur cette définition, il y avait 217 lois spéciales à caractère régional, soit un peu plus de $10 \%$ des 1904 articles additionnels présents dans le code à la fin des Qing. Leur répartition au sein des sept parties de ce code est indiquée au tableau 1. Parmi ces articles additionnels, six concernent quasiment l'ensemble des provinces, cinq les territoires occupés par les Miao et autres populations non-Han, trois concernent le cours du fleuve Jaune et le grand canal, deux sont intitulés « hors les passes » ce qui désigne la Mandchourie, six ont pour objet des régions littorales ou des montagnes reculées. Les 197 articles restants se rapportent à des provinces ou lieux clairement déterminés : parmi eux, vingt et un concernent deux provinces ; douze concernent trois provinces ; trois concernent quatre ou cinq provinces ; quatre concernent six provinces, soit un total de 283 mentions de provinces. Un dernier article porte sur une province mais aussi sur les régions littorales, le cours du fleuve Jaune et le grand canal. Les statistiques propres à chaque province sont indiquées au tableau 2.

5. Xue $1970: 2195$ (Art. 427-05). 


\begin{tabular}{|c|c|c|c|c|c|c|c|c|}
\hline 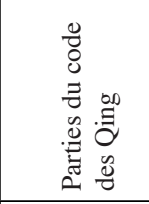 & 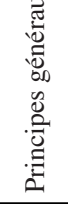 & 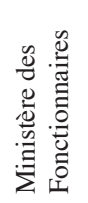 & 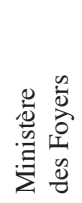 & 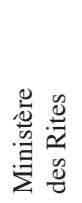 & 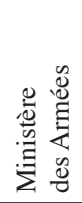 & 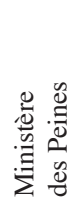 & 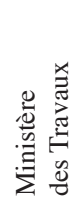 & 离 \\
\hline \begin{tabular}{|l|l|} 
Nombre \\
d'articles \\
additionnels \\
\end{tabular} & 223 & 75 & 289 & 52 & 152 & 1087 & 26 & 1904 \\
\hline
\end{tabular}

Tableau 1. Répartition des lois régionales dans les sept parties du Code des Qing

\begin{tabular}{|c|c|c|c|c|c|c|c|c|c|c|c|c|c|}
\hline Circonscriptions administratives & 表 & 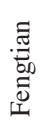 & 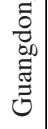 & 恵 & 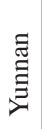 & : & 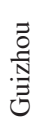 & 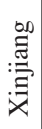 & క & 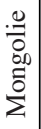 & 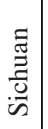 & 毒 & 烝 \\
\hline Nombre de lois spéciales & in & ¿ & 2 & $=$ & 눈 & 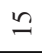 & \pm & \pm & $\simeq$ & $=$ & $=$ & 으 & 운 \\
\hline Circonscriptions (suite) & 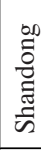 & $\begin{array}{l}\text { 㞼 } \\
\end{array}$ & $\begin{array}{l}\overrightarrow{0} \\
\stackrel{\Xi}{\Delta} \\
\end{array}$ & 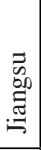 & 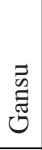 & 寻 & 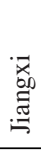 & 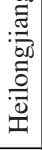 & है & 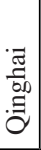 & $\begin{array}{l}\vec{x} \\
\bar{\Xi} \\
\text { ज्ञ }\end{array}$ & 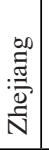 & \\
\hline Nombre de lois spéciales & 9 & 8 & 6 & 6 & 5 & 5 & 4 & 3 & 3 & 2 & 2 & 1 & \\
\hline
\end{tabular}

Tableau 2. Nombre de lois spéciales par province dans le Code des Qing

Sous la dynastie des Qing, les articles originaux du code hérités des Ming restèrent fondamentalement les mêmes, tandis que des articles additionnels étaient ajoutés ou abrogés à tout moment, en vertu de révisions périodiques qui adaptaient le code au cours des événements. Les lois spéciales à caractère régional étaient en général compilées à la demande des gouverneurs de province, mais devaient obtenir l'agrément des instances dirigeantes suprêmes avant d'être codifiées sous forme d'article additionnel. Vues sous l'angle des institutions juridiques, ces lois manifestent les relations entre le pouvoir suprême et le gouvernement local ainsi que les différences d'appréciations à propos du maintien de la paix et de l'exercice de la justice au niveau local. Ils révèlent donc la vision stratégique qu'avaient les Qing de la géographie politique de leur empire et des nécessités d'y adapter leur système juridique. 


\section{Contexte institutionnel}

La Chine des Qing représente un cas unique par sa durée et la superficie du territoire contrôlé. À l'intérieur d'un pays aussi vaste et composé de peuples si nombreux, la géographie naturelle et humaine de chaque lieu était différente, et une législation nationale unifiée ne pouvait pas prendre en compte l'ensemble des problèmes propres à chaque région. La mise en œuvre de législations et de cours de justice locales était inévitable. Mais dans la conception traditionnelle, « le droit est l'émanation du prince » (fa zi jun chu). Cette idée d'une concentration du pouvoir législatif était fermement ancrée, le souverain étant nominalement l'unique législateur représentant le Centre de l'univers normatif. « Le prince est le droit vivant ${ }^{6} »$, « les rites, la musique et l'ordre de partir en guerre viennent du Fils du $\mathrm{Ciel}^{7} »$, le Fils du Ciel « unifie le gouvernement du monde ${ }^{8} »$, de sorte que « partager le droit de punir et de récompenser reviendrait à dissoudre l'autorité ${ }^{9} »$ : on voit que tous les penseurs de la période précédant l'unification impériale par le Premier empereur des Qin se sont faits l'écho de ces principes. Les dynasties se sont succédé, mais la concentration du pouvoir législatif ne fut jamais remise en cause. Et ce quand bien même des critiques acerbes visèrent la concentration des pouvoirs ou prônèrent leur séparation. Ainsi, Huang Zongxi (1610-1695), penseur éclairé du début des Qing, émit dans son Plan pour le prince (Mingyi daifang $l u)$, des propos favorables à l'autonomie, comme par exemple : "lorsque le général est seul au commandement, il supporte seul les fautes », «les biens produits en un endroit doivent être disponibles en cet endroit», ou encore « les troupes originaires d'un certain lieu doivent servir en ce lieu $»^{10}$. C'est à partir des Song (960-1279) que la concentration des pouvoirs et les institutions politiques correspondantes se développèrent parallèlement, conformément à l'esprit selon lequel il faut « se préparer aux difficultés et mettre en place les institutions pour y faire face ${ }^{11} »$. Ce mouvement connut son apogée sous les Qing. Alors, le système associant « trois degrés de circonscriptions administratives à cinq degrés de gouvernement ${ }^{12}{ }^{2}$ témoignait de la volonté de

6. Guanzi 45.4, (Renfa 任法).

7. Lипуи 16.2 (Jishi 季氏)

8. Mozi $3.1($ ( 6)

9. Hanfeizi $48(\S 1)$

10. Huang $1981: 21-22$.

11. Hanshu (Liyuezhi 禮樂志).

12. NDT. « Trois degrés de circonscriptions administratives », c'est-à-dire la province (sheng), la préfecture $(f u)$ et le district ou sous-préfecture (xian) ; "Cinq degrés de gouvernement », c'est-à-dire : 1. le gouverneur général à la tête de plusieurs provinces 
l'empereur d'un contrôle par le centre et d'une surveillance mutuelle entre les fonctionnaires locaux, afin de consolider un plan gouvernemental privilégiant le centre au détriment de la périphérie (nei zhong wai qing) ${ }^{13}$. Si la bureaucratie locale devenait trop puissante, le pouvoir central risquait de ne plus pouvoir la contrôler. Il fallait donc priver les fonctionnaires locaux d'une partie de leurs prérogatives administratives et veiller à ne pas leur déléguer le pouvoir législatif inconsidérément. D'une part, il y avait donc un besoin objectif de législation locale, mais de l'autre, le pouvoir des gouvernements locaux, en particulier sur le plan législatif, était limité et ne pouvait s'exercer que sous la forme de lois spéciales qui ne pourraient être promulguées que par l'administration centrale pour une région donnée. Cette catégorie juridique, assez rare dans les institutions politiques modernes, était très développée à l'époque et son rôle était manifeste. C'est là le signe d'un haut degré de centralisation du pouvoir.

Ce n'était pas là une innovation des Qing. Des dispositions spéciales sur la protection de la capitale existaient déjà dans le code des Tang, bien qu'en nombre très réduit. C'est à partir des Song que de nombreuses lois spéciales à caractère régional apparurent. Les chiffres parlent : quatre éditions des «Décrets, ordonnances, règlements et formulaires » (Chi ling ge shi) en vigueur dans la région de Pékin furent publiés, tandis que les décrets sur les districts, préfectures et circuits furent promulgués à sept reprises, à quoi s'ajoutent encore quatre éditions de lois spéciales sur des lieux stratégiques ${ }^{14}$. Le «Traitéjuridique (Xingfa zhi) de l'Histoire officielle des Song (Song shi) indique que sous le règne de Renzong (1023-1064), les ordonnances propres aux circuits, préfectures et districts atteignirent le nombre de 3300. Les fameuses « lois sévères sur les vols et brigandages » (daozei zhongfa) étaient sans doute du nombre. Les nombreuses lois à caractère régional compilées à ce moment sous la forme de recueils d'ordonnances sont très semblables à celles qui furent plus tard insérées sous forme d'articles additionnels dans le code des Qing. Les Song furent une époque de concentration des pouvoirs, dont la pensée directrice était que «le centre est plus important que la périphérie » (nei zhong wai qing). Ces similitudes ne sont donc pas le fait du hasard car, dans une certaine mesure, les institutions plus tardives ont leur origine dans une tradition de centralisation des pouvoirs législatifs et politiques remontant aux Song.

(zongdu), 2. le gouverneur de province (xunfu), 3. l'intendant de circuit (daotai ou daoyuan), 4. le préfet (zhifu, zhizhou), 5. le magistrat local ou sous-préfet (zhixian).

13. Zhou 1998 : chapitre 8 .

14. Guo $1997: 31$. 
C'est sous les Qing que les articles additionnels devinrent la forme privilégiée des lois spéciales à caractère régional dans un système juridique centralisé. À partir de l'ère Qianlong (1735-1796), les articles originels (lii) du code des Qing ne furent plus amendés ni révisés. Comme ces articles jouaient le rôle stabilisateur de loi fondamentale, ils renferment très peu de règles spéciales adaptées à des circonstances locales particulières. Seulement huit de ces articles sur un total de 436 ont la qualité de règles locales ${ }^{15}$, soit moins d' $1 / 50^{\mathrm{e}}$, et ils se rapportent principalement à Pékin ${ }^{16}$. Les articles additionnels à caractère régional sont en bien plus grand nombre. L'ajustement des règles pénales aux besoins du moment se faisait sous les Ming et les Qing par la codification d'articles additionnels (tiaoli). Les nouveaux articles étaient formellement promulgués par l'empereur, mais la grande majorité d'entre eux étaient des mémoires soumis par les fonctionnaires de l'administration centrale ou par les gouverneurs provinciaux. Leur contenu était donc conforme au mémoire ratifié par l'empereur, moyennant d'éventuels ajouts ou modifications. Il est dès lors naturel de trouver des règles relatives aux régions frontières, déterminées en fonction des circonstances par les fonctionnaires envoyés dans ces territoires. Parmi les 217 articles spéciaux à caractère régional, au moins 121 ont été suggérés par des gouverneurs de province, des trésoriers ou juges provinciaux qui les assistaient, par des Surintendants au Qinghai et en Mongolie, ou par le Préfet de Shuntian (la préfecture qui couvrait Pékin) ou les administrateurs de Shengjing-Moukden, la première capitale mandchoue : c'est-à-dire que ces 121 articles additionnels émanent de l'administration territoriale, tandis que 35 seulement sont manifestement issus d'organes centraux comme le ministère des Peines, la Cour administrant les provinces extérieures ou le Censorat. Ces lois spéciales régionales sont le produit législatif naturel des mémoires soumis par les fonctionnaires de l'administration territoriale en fonction des problèmes spécifiques qu'ils rencontraient sur le terrain.

Sous les Ming, le nombre d'articles additionnels était encore limité. Les Règlements sur l'instruction des affaires pénales (wenxing tiaoli) de l'ère Hongzhi (1488-1506) n'en contenaient que 279 et ceux de l'ère Jiajing (15221567) ne dépassaient pas 385 . Les lois spéciales étaient aussi en faible nombre, parmi lesquelles on trouve des lois régionales comme celle-ci :

15. Deux de ces articles se trouvent dans la partie dite des «noms et règles » (mingli), quatre dans la partie «militaire » (bingbu), et deux dans la partie «pénale » (xingbu).

16. Voir les articles 198,217,219,223 dans le chapitre sur les lois militaires et l'article 261 dans le chapitre sur les lois pénales. 
Au Henan, faire des brèches dans les digues soit clandestinement soit délibérément, provoquant ainsi des nuisances pour les habitants, la perte de biens, l'ennoiement des récoltes, toutes fautes passibles d'une peine supérieure à la servitude : pour l'auteur principal, si c'est un suppléant dans une bannière ou un civil : exil militaire dans une région proche ; si c'est un militaire : mutation dans une garnison frontière ${ }^{17}$.

Cette disposition fut reprise sous les Qing moyennant quelques ajouts et modifications ${ }^{18}$. Le nombre d'articles additionnels augmenta rapidement sous les Qing, pour avoisiner les 2000 à la fin de la dynastie. Très détaillés dans leur contenu, ils adaptaient le sens des anciens articles aux temps et aux lieux, si bien qu'ils devinrent la principale source du droit et la forme essentielle des lois spéciales à caractère régional.

\section{Contenu des lois spéciales à caractère régional}

Le contenu de ces lois peut être divisé en deux grandes catégories : les dispositions substantielles et les dispositions procédurales. La fonction essentielle des dispositions substantielles est de qualifier les crimes et de fixer les peines correspondantes, tandis que les dispositions procédurales règlent les questions relatives à l'arrestation, l'enquête, le jugement et l'exécution des décisions. Les lois spéciales à caractère régional, tant dans leurs dispositions substantielles que procédurales, visaient principalement les crimes les plus graves, et leur caractéristique la plus générale était d'aggraver les peines prévues par les lois communes.

La concentration de crimes d'une grande malfaisance en un même lieu menaçait directement la stabilité sociale et l'ordre impérial. Aussi cela fitil constamment l'objet de mesures de répression particulièrement sévères. Comme l'indique la maxime "l'administration du royaume n'a pas de plus grande urgence que vols et brigandages ${ }^{19} »$, ces deux crimes représentaient le type même de la malfaisance. Dans la partie «pénale » du code des Qing qui rassemble les «Lois du ministère des Peines » (Xinglü, voir le tableau 1), c'est la section «Vols et Brigandages » qui comporte le plus grand nombre d'articles additionnels et qui couvre les crimes les plus graves. C'est aussi dans cette section qu' on trouve le plus grand nombre de lois spéciales à caractère régional,

17. Mingdai tiaoli.

18. Xue 1970 : 1303 (Art. 433-01). L'article fut modifié en 1804 et 1820.

19. Fang $1974: 922$. 
soit 63 . Ce chiffre représente $70 \%$ des 91 lois spéciales à caractère régional contenant des dispositions substantielles dans la partie «Lois pénales ».

Pour autant, 47 de ces lois spéciales comportent des dispositions substantielles comparables à celles qu'on trouve dans une loi générale de même nature mais valide sur tout le territoire. Il est donc intéressant de les comparer. 38 de ces lois générales prévoient des peines égales ou supérieures à la servitude pénale, 34 prévoient des peines égales ou supérieures à l'exil, dont la moitié de peines capitales. C'est dire que la plupart des actes criminels punis par ces lois générales sont déjà assez graves, et leur sanction plutôt sévère. Pourtant, les lois spéciales à caractère régional ajoutent encore en gravité, comme les provinces du Guangxi et du Guangdong en offrent un bon exemple. Passible de servitude pénale dans les lois générales, l'enlèvement de plus de trois enfants suivi de plus de trois demandes de rançon y était passible de l'exil militaire à $4000 \mathrm{li}$ selon les lois spéciales ; lorsque la peine générale était l'exil militaire ou l'exil servile, la peine spéciale à la région était la strangulation sous réserve de révision par les Assises d'automne ; de la même manière, les peines de mort soumises à révision dans les lois générales étaient prononcées «d'exécution immédiate » dans les lois spéciales ${ }^{20}$.

De fait, lorsqu'on compare les 47 articles mentionnés plus haut à la loi générale correspondante, un seul se présente dans l'ensemble moins sévère que la loi générale ; quatre articles sont moins sévères pour une partie seulement de leurs dispositions, un seul article est aussi sévère que la loi générale, et cinq autres sont d'un même degré de sévérité, bien qu'une partie de leur contenu diffère assez de la loi générale. Toutes les autres lois spéciales à caractère régional sont plus sévères que les normes appliquées sur l'ensemble du territoire. Du fait que l'objectif essentiel des dispositions substantielles était la lutte contre les crimes graves, le pouvoir impérial a ouvertement recouru à la sanction plutôt qu'à l'éducation, et ceci depuis l'institutionnalisation sous les Song des «territoires aux lois sévères » (zhongfadi). Conformément aux enseignements des textes anciens tels que " les sanctions pénales sont plus ou moins sévères selon l'époque ${ }^{21}$ » et « les temps troublés nécessitent l'usage de peines sévères ${ }^{22}{ }$, les lois spéciales à caractère régional étaient habituellement plus sévères que les lois générales. Dans l'Édition conjointe des codes des Tang et des Ming (Tang Minglü hebian) où il compare article par article le code des Tang à celui des Ming, Xue Yunsheng remarque que le droit de cette dernière dynastie se singularise par « l'aggravation des peines sévères

20. Xue $1970: 718$ (Art. 273-18).

21. Shangshu.

22. Zhouli. 
et l'allègement des peines clémentes ${ }^{23} »$. Les Qing ayant hérité du droit des Ming, cette remarque visait en réalité les institutions de la dynastie régnante. La sévérité des Qing à l'égard des crimes graves trouve certainement ici son explication.

Les dispositions procédurales contenues dans les lois spéciales réglaient principalement l'établissement des dossiers sur les cas d'homicides et de vols commis à la capitale, ainsi que les procédures d'enquête et d'exécution des peines de servitude pénale ou d'exil dans les provinces. Les fonctionnaires locaux n'avaient de juridiction propre (zili cisong) que sur des cas criminels véniels ou des affaires civiles pour lesquelles il n'y avait pas de chef d'accusation précis dans le code, toutes affaires qui n'étaient passibles que de bastonnades. Pour les cas passibles de la servitude pénale ou d'une peine plus sévère, les magistrats locaux devaient procéder à une première instruction et émettre une proposition de jugement, puis la transmettre à la capitale provinciale pour le jugement final. Les peines de servitude étaient instruites et jugées par le gouverneur de province, les peines d'exil par le ministère des Peines. Les jugements proposant la peine capitale devaient être rapportés à l'administration centrale, et lorsque la peine était soumise à révision par les Assises d'automne, c'était le pinceau impérial qui donnait l'autorisation d'exécuter.

Afin de tenir compte des situations spécifiques propres à chaque région, les Qing promulguèrent des règles relatives à l'instruction des affaires graves adaptées aux différents contextes. Par exemple, des articles dispensaient des préfectures ou districts trop éloignés de la métropole provinciale d'y transférer les prisonniers au moment où leur cas était revu dans le cadre de la procédure des Assises d'automne. Autre exemple, un article additionnel annexé en 1748 à la loi sur les degrés de compétence des tribunaux pour l'exécution des condamnations envisage diverses solutions pour remplacer un magistrat qui ne peut présider aux condamnations capitales dans son district :

Lorsqu'il faut procéder à des exécutions capitales dans la province du Yunnan, si à réception de l'ordre d'exécuter envoyé par le ministère des Peines le magistrat titulaire est parti en mission et qu'il n'y a pas de fonctionnaire adjoint (zuo'er) au chef-lieu de district, - mis à part le cas où le préfet a été informé à temps de la mission du magistrat titulaire et que, le district de ce dernier étant dans les proches environs de la préfecture, le préfet a pu conformément au règlement déléguer un chargé de mission pour surveiller l'exécution - dans les autres cas où le district n'est pas dans les proches environs de la préfecture, ou bien si le magistrat a été muté

23. Xue $2000: 170$. 
subitement et n'a pu prévenir le préfet à temps, dès réception de l'ordre d'exécuter envoyé par le ministère, le chef du secrétariat, le responsable de la prison, le chef de brigade sont autorisés à superviser ensemble l'exécution par procuration.

Selon les Commentaires de M.Xue, l'article s'explique par le fait que la plupart des districts du Yunnan manquaient d'un fonctionnaire adjoint susceptible de remplacer le magistrat titulaire ${ }^{24}$. Selon le droit commun, en cas d'absence du sous-préfet, la surveillance des exécutions aurait dû être confiée au fonctionnaire adjoint, ou à défaut au substitut que le préfet aurait dû envoyer. Mais comme en général aucun adjoint n'était disponible, il fallut adapter ces règles en autorisant un groupe de subalternes à remplacer le fonctionnaire défaillant.

Les lois spéciales à caractère régional concernaient principalement les crimes les plus graves, d'où l'étroite interrelation entre la structure centralisée du système judiciaire et les modes de délégation de pouvoir. Le système de révision plaçait les tribunaux inférieurs sous l'étroit contrôle des échelons supérieurs dès que les premiers prononçaient une peine égale ou supérieure à la servitude. Les magistrats locaux disposaient d'une marge de liberté importante pour juger les affaires mineures, mais les jugements prononçant des peines égales ou supérieures à la servitude devaient être strictement conformes au droit codifié $^{25}$. Les affaires relevant de la juridiction propre des magistrats étaient souvent, en raison de la pauvreté des moyens dont ils disposaient, considérées comme ne relevant pas d'un procès stricto sensu, alors que l'administration centrale évitait de s'en occuper et les laissait donc au ressort du magistrat local.

Pour autant, l'administration centrale ne pouvait interférer dans toute affaire locale, quelle qu'en soit l'importance, en privant le gouvernement local de tout pouvoir législatif. Dans le cadre des institutions traditionnelles, les magistrats locaux avaient la compétence d'interpréter et d'adapter les normes de l'administration centrale et d'exercer, au nom de leurs compétences administratives, une part du travail législatif. Les proclamations (gaoshi) et les prohibitions (jinyue) étaient la forme principale utilisée par les magistrats pour exercer leur «gouvernance locale » (tu zhengce). Il n'était pas rare que ce type de documents soit utilisé pour compléter les insuffisances des lois. Il arrivait même aux magistrats d'adapter ainsi les règles du code. Par exemple, une proclamation émise par un magistrat local avertissait les justiciables que : «Dans les plaintes, il n'est pas permis de mélanger des affaires datant d'années anciennes, ou qui ont été amnistiées, pour concocter des accusations

24. Xue $1970: 1258$ (Art. 411-40).

25. Shiga 1986. 
criminelles ; les plaintes qui le font quand même ne seront pas prises en considération ${ }^{26}$. Or, selon l'article additionnel 16-3 du code des Qing, hérité des Ming et annexé à l'article « Des crimes ne pouvant être pardonnés par une amnistie », quelqu'un qui dénonçait autrui pour un crime en sachant pertinemment que celui-ci avait été amnistié devait subir la peine encourue par celui qu'il avait dénoncé ${ }^{27}$. S'agissant d'affaires d'argent, de grains, et autres affaires civiles rencontrant une amnistie, une enquête minutieuse devait montrer comment statuer en l'absence d'indication précise sur le degré de peine. La proclamation du magistrat local, menaçant tout au plus de ne pas prendre en compte la dénonciation d'un crime amnistié, était donc en contradiction avec l'article du code, beaucoup plus sévère. De fait, ces proclamations et prohibitions avaient une tournure très personnelle et n'avaient souvent de valeur que du vivant de leur auteur.

Un tel phénomène pouvait également prendre la forme des « règlements provinciaux » (shengli). Selon les règlements édités sous les Qing qui nous sont parvenus, il en existait cinq ou six espèces ${ }^{28}$. Si l'on prend l'exemple des Règles établies de l'administration du Zhejiang (Zhi Zhe chenggui), une bonne partie était constituée de documents transmis par le Trésorier ou le Juge provincial au gouverneur pour que celui-ci statue, ce sont donc des règlements de nature administrative. Mais d'autres règles assez nombreuses étaient de nature législative et n'étaient pour certaines que la mise en application des règles formulées par l'administration centrale. Quelques-unes adaptaient les règles générales en se fondant sur un examen attentif de la situation locale. Toutes étaient applicables à l'ensemble de la province du Zhejiang et avaient un caractère pérenne, ce qui est le propre d'une législation locale.

Cependant, qu'il s'agisse de décisions judiciaires prises par le magistrat sous sa juridiction propre, des proclamations et prohibitions ou des règlements provinciaux, toutes ces règles n'étaient applicables qu'aux affaires mineures. La situation était différente pour les affaires graves, en particulier celles passibles d'une peine supérieure ou égale à l'exil. Les hauts fonctionnaires en poste aux frontières ou les gouverneurs de province devaient transmettre ces affaires au ministère des Peines dont les directeurs, bien que leurs égaux en grade, décidaient de l'exécution des peines d'exil, ce qui montre bien la nature centralisée des institutions. Les fonctionnaires locaux souhaitant accroître la répression à l'encontre de certaines catégories de crimes pour tenir compte de circonstances locales, ou encore modifier la procédure afin d'adapter les

26. Wu 1999: 220 (juan 5)

27. Xue $1970: 48$ (Art. 16-03).

28. Terada 1993. 
modes de contrôle aux réalités, étaient restreints par les règles codifiées. Ils ne pouvaient se fier à leur propre initiative ni, à plus forte raison, fixer leurs propres règles. Comme nous l'avons déjà indiqué, la majorité des lois régionales spéciales furent promulguées à la suite de mémoires soumis par les gouverneurs provinciaux. Dans une telle situation, toute adaptation devait faire l'objet d'une demande circonstanciée qui devait recevoir l'accord de l'empereur avant d'être formalisée selon les règles de la législation centrale et mise en vigueur.

\section{Répartition spatiale des lois spéciales à caractère régional}

Dans un système si centralisé, il est évident que toutes les demandes locales ne pouvaient être satisfaites. Le choix des régions où seraient appliquées des dispositions spéciales se faisait du point de vue du gouvernement central. En analysant la répartition des articles contenant des dispositions spéciales à caractère régional, on peut s'apercevoir que celle-ci n'est pas homogène mais répond à certains principes. La carte 1 montre que le point focal du dispositif était Pékin. Ensuite, le berceau de la dynastie, la région du Fengtian, rattachée à la capitale secondaire de Shengjing, faisait également l'objet d'une attention toute particulière. Les articles additionnels relatifs à ces deux régions occupent plus de $2 / 5$ du total.

Un deuxième groupe comprend neuf provinces pour lesquelles on trouve plus de dix articles contenant des dispositions spéciales à caractère régional. À l'exception du Zhili, la province métropolitaine autour de Pékin, ces provinces couvrent la totalité des frontières du Nord et du Sud de la Chine, régions souvent occupées par des populations non Han et éloignées du centre. Les 14 provinces ou territoires restants font l'objet de 10 articles ou moins, et ne connaissent que des écarts très faibles.

Les articles additionnels relatifs à la capitale, les plus nombreux, aggravaient les peines pour les crimes les plus malfaisants. Le vol avec violence était un crime particulièrement réprimé en droit chinois et la tendance fut à l'aggravation des peines entre les Tang et les Qing. Mais lorsque les faits se déroulaient à Pékin, la peine était encore plus sévère. Par exemple, lorsqu'un criminel était armé ou s'était introduit dans une maison, mais sans obtenir de butin ni blesser personne, la peine était l'exil militaire à $3000 \mathrm{li}$ dans le droit des Tang. Au début des Qing, la peine pour des faits identiques fut aggravée en déportation au Xinjiang avec réduction en esclavage. Un article additionnel de 1852 condamnait désormais l'auteur principal à la strangulation après 
révision aux Assises d'automne ${ }^{29}$. De la même façon, les sanctions à l'égard des fonctionnaires n'arrêtant pas les voleurs dans les délais légaux étaient aggravées lorsque ceux-ci étaient en poste à la capitale. Des constatations similaires peuvent être faites à l'égard des articles spéciaux mis en vigueur à Shengjing, la capitale mandchoue.

Les aires métropolitaines de Pékin et Shengjing avaient aussi des dispositions particulières concernant l'exécution des peines. Par exemple, un article additionnel annexé en 1801 à l'article intitulé « Lieux d'exil, de servitude et de relégation » disposait que : «Les civils condamnés à la peine de servitude à Pékin doivent être envoyés dans un district ou une préfecture situés à plus de $500 l i$ de la capitale pour y purger leur peine ${ }^{30}$.» Un autre article indiquait les règles à suivre dans le choix du lieu de destination des condamnés à l'exil ou à l'exil militaire «qu'il était malséant d'installer » dans les provinces du Zhili et du Fengtian entourant les deux capitales. De telles règles visaient au maintien de l'ordre public dans ces deux régions sensibles.

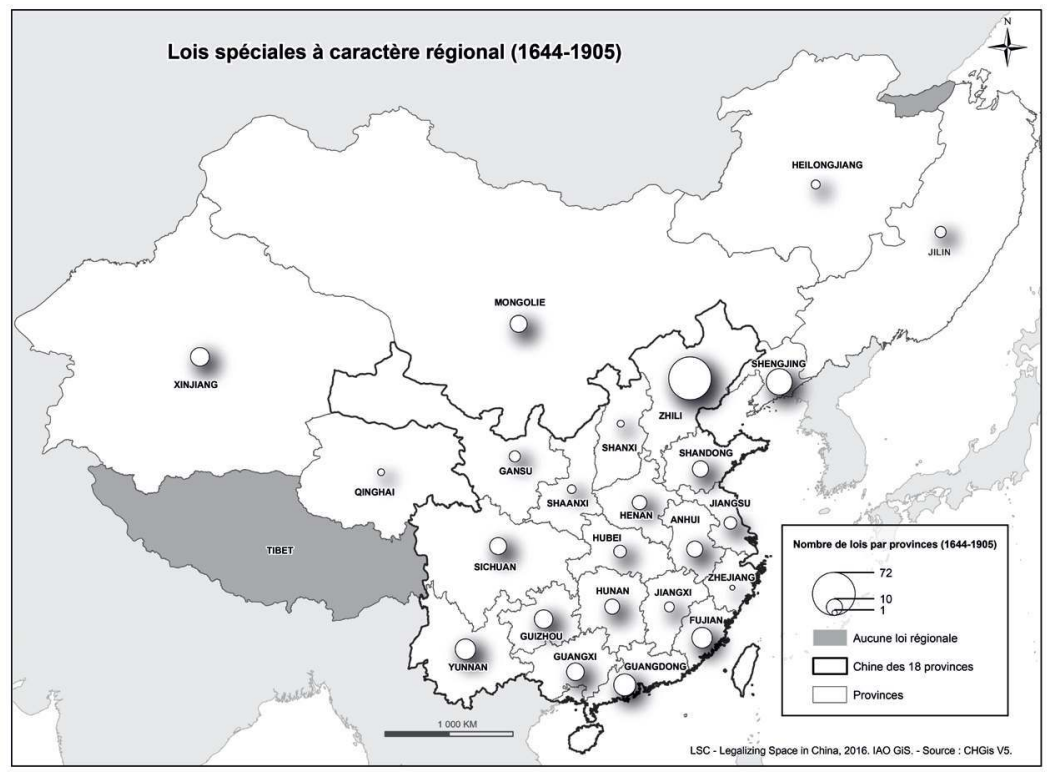

Carte 1. Répartition territoriale des lois spéciales à caractère régional

29. Xue $1970: 619$ (Art. 266-44).

30. Xue $1970: 150$ (Art. 45-10). 
Les régions frontières faisaient l'objet de nombreuses dispositions spéciales à caractère régional car elles couvraient la plupart des territoires occupés par des populations non-Han. Les Qing adoptèrent plusieurs législations spécifiques aux Mongols, Ouïghours et Tibétains pour tenir compte des spécificités de leurs coutumes, à l'instar du Code mongol, des Règlements $d u$ Turkestan ou encore des Règlements de Xining pour l'administration des Tibétains. Certaines de ces règles à caractère local sont devenues des articles additionnels du code des Qing. Par exemple, celui qui fut annexé en 1818 à l'article 34 : «Crime commis par un étranger à l'empire » indique que pour les infractions commises en Mongolie, on applique aux criminels mongols le Code mongol, et aux criminels chinois le Code des Qing ${ }^{31}$. En cas de coaction entre criminels mongols et chinois, on applique le texte dont les dispositions sont les plus sévères. Ce genre de dispositions visait au maintien de l'ordre et de la stabilité sociale dans des conditions propres à chaque lieu. De fait, les zones frontalières étaient souvent des territoires propices à des criminalités particulières, voire même à des révoltes populaires de grande ampleur. Ainsi, on trouve quatre dispositions spéciales régionales visant plus particulièrement l'enlèvement pour exiger une rançon dans la région du Guangdong, indiquant sans doute que la pratique y était courante ${ }^{32}$.

Outre les régions frontières, les régions de forte criminalité faisaient aussi l'objet de lois spéciales. Wang Fengsheng (1776-1834), qui fut le préfet de Guide au Henan dans les années 1820, indiquait dans un manuel à l'adresse des fonctionnaires que les territoires de sa préfecture limitrophes de l'Anhui et du Shandong connaissaient une criminalité endémique ${ }^{33}$. Ces lieux apparaissent régulièrement dans les articles additionnels du code. L'un d'entre eux mentionne expressément les préfectures de Guide (Henan), Yingzhou et Fengyang (Anhui) à propos de violences collectives entre travailleurs à gages, contre lesquelles une peine aggravée était prévue ${ }^{34}$.

La répartition spatiale des lois spéciales à caractère régional était guidée par la volonté du législateur de protéger la capitale au centre tout en conservant aux régions frontières leur rôle de bouclier. Si la sécurité de Pékin a constamment revêtu la plus grande importance, c'est sous les Qing que la région de Shengjing, en tant que territoire d'origine et base arrière des Mandchous, a eu une place particulière et similaire à celle de Pékin. En raison du centralisme et de l'étendue

31. Xue $1970: 133$ (Art. 34-03).

32. L'information est confirmée dans la Monographie du district de Dongguan. Cf. Dongguan xian zhi, juan 9.

33. Wang $1997: 372$.

34. Xue 1970 : 644 (Art. 268-23), 717 (Art. 273-17) et 895 (Art. 302-09). 
de l'empire, les régions frontières constituaient le maillon faible du pouvoir. Parmi elles, le Guangxi, le Guangdong, le Guizhou, le Yunnan au sud-ouest et le Turkestan au nord-ouest étaient les destinations des condamnés à la peine « d'exil sur une frontière très éloignée », « dans une région insalubre » ou de la « grande déportation avec réduction en esclavage ». Ces territoires étaient considérés comme arriérés et habités par des Barbares qui avaient des coutumes bien différentes de celles promues par la cour impériale. Par exemple, dans un mémoire soumis en 1735, Feng Guangyu, alors commissaire aux finances du Guizhou, indiquait que « les Miao ne connaissent ni rites ni loi, leur règle est que tout meurtre ou toute blessure se solde par un certain nombre de têtes de bétail. Le fort dévore le faible, puis on transige pour un certain poids de grains [...] ». D'ailleurs, ajoutait-il, impossible d'enquêter et d'arrêter des suspects sans que tout le voisinage vienne à la rescousse ${ }^{35}$. Lan Dingyuan (1680-1733) fit des remarques similaires après la campagne qu'il mena à Taiwan sur ordre de Kangxi en $1721^{36}$. Quelques rares régions de Chine intérieure mises à part, une bonne partie des crimes commis l'étaient dans ces marges frontières. Sous les Qing, de nombreuses révoltes se produisirent dans les territoires occupés par des populations non Han ; à Taïwan, dans le Sud-Ouest ou le Nord-Ouest de la Chine, la volonté d'autonomie rendait la situation encore plus complexe et obligeait le gouvernement à rester sur ses gardes. Alors que, dans des provinces intérieures comme le Jiangxi et le Zhejiang, l'économie et la culture étaient florissantes et le maintien de l'ordre assuré, renforcer le contrôle sur les régions frontières devint une question cruciale pour la stabilité de l'empire.

En résumé, la production, le contenu et la répartition de ces lois spéciales eurent pour fondement la concentration du pouvoir politique au sein de l'administration centrale. Sous un tel régime, elles jouèrent un rôle non négligeable dans l'aménagement des relations entre le centre et la périphérie, sans oublier la distribution appropriée des pouvoirs sur le territoire, ainsi que le maintien de l'ordre dans la capitale et la stabilité aux frontières.

35. Qingdai nongmin zhanzheng shi ziliao xuanbian : tome 3,60-61. Voir l'article de Zhang Ning dans ce volume.

36. Lan Dingyuan, Taiwan baojia ze chengxiang changshu. 


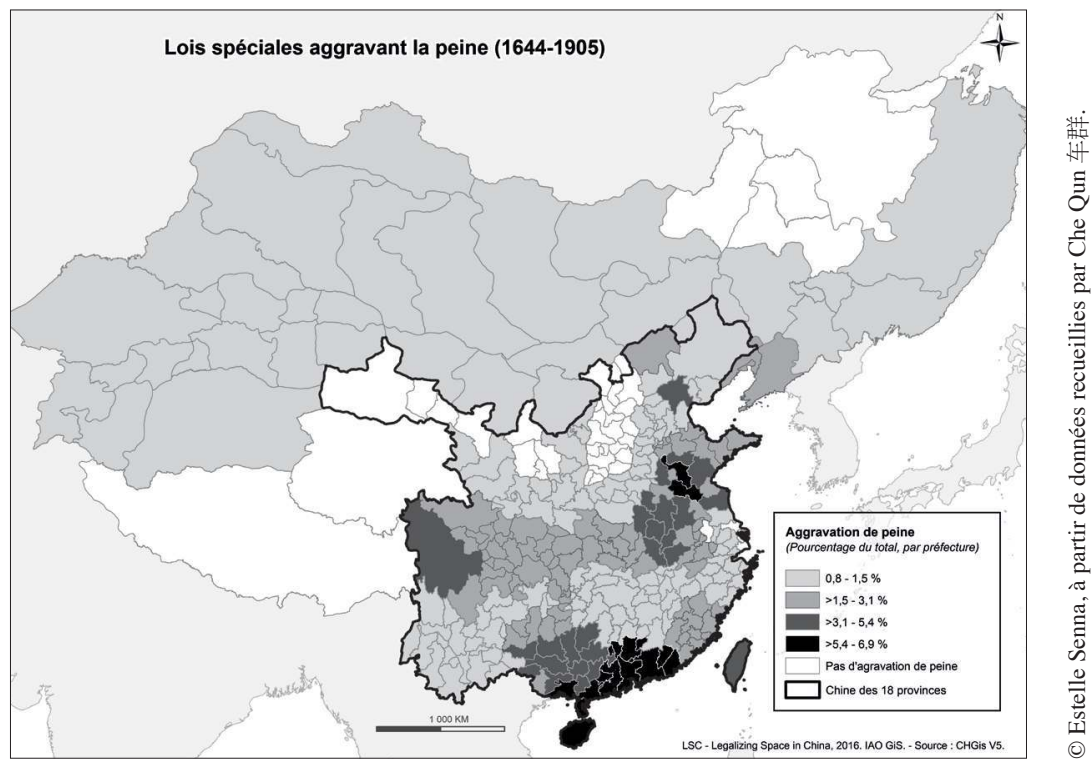

Carte 2. Des lois plus sévères pour les zones sensibles

\section{Des lois spéciales aux lois générales}

Selon Montesquieu, le droit est censé correspondre aux conditions naturelles, au climat, au territoire, aux conditions de vie de la population, au degré de liberté politique, ainsi qu'à la religion, la richesse, la démographie, le commerce et les mœurs ${ }^{37}$. Même si la société chinoise traditionnelle pratiquait la centralisation en matière législative et judiciaire, des spécificités propres à chaque lieu influençaient la législation et rendaient impossible toute uniformisation. D'où le recours à des législations à caractère local qui, sous les Qing, prirent la forme d'articles additionnels. Ces lois visaient à un meilleur équilibre des pouvoirs central et local, elles contribuaient à la protection du pouvoir impérial, à la lutte contre la grande criminalité, à la préservation des relations entre populations ethniques différentes, ainsi qu'à la stabilisation des régions frontières. Certaines de ces lois préservaient en outre l'équité et l'efficacité du système pénal. Par exemple, la peine d'exil établie comme l'une des cinq peines légales depuis l'époque des Qi (550-577) jusqu' aux Qing joua toujours un rôle majeur dans le système pénal. Comme chaque province avait

37. Montesquieu $1834: 29$. 
sa situation géographique propre, les condamnés à l'exil subissaient leur peine dans des lieux différents. Afin de conserver l'égalité et l'équité des peines, un article du code déterminait le lieu ou un condamné à l'exil devait subir sa peine en tenant compte de son lieu de départ. Des articles additionnels vinrent actualiser ce principe, de sorte que l'exécution de la peine se fît dans des conditions d'égalité et d'équité maximale ${ }^{38}$.

Pourtant, l'accroissement du nombre d'articles additionnels dû à la concentration des pouvoirs et aux lacunes de la technique législative fit apparaître les inconvénients du système. La plupart de ces lois procédaient de mémoires présentés par les gouverneurs provinciaux à la ratification de l'empereur. Elles manquaient donc trop souvent d'une vision d'ensemble, elles ne tenaient compte que de l'intérêt local et de l'objectif poursuivi, provoquant des excès de sévérité ou de clémence contraires à la justice. Ainsi, l'article additionnel 273-16 annexé à l'article intitulé « Extorsion de biens par intimidation » dispose que :

Les brigands de la province du Guangdong qui pénètrent secrètement dans la province du Guangxi pour s'acoquiner avec les brigands locaux, former une confrérie jurée entre criminels, piller, escroquer, etc., commettent un crime passible de la peine d'exil militaire ou d'une peine inférieure : en vertu du présent article, aggraver la peine d'un degré.

Comme l'article avait son origine dans un mémoire soumis par le juge provincial du Guangxi, il était manifestement établi pour maintenir l'ordre public dans cette province. Cependant, comme l'indiquent les Commentaires de M. Xue : «Il n'y a aucun article spécifique concernant la réunion entre criminels, le pillage, l'escroquerie dans la province du Guangdong [...] et c'est seulement lorsque des criminels pénètrent clandestinement au Guangxi qu'il y a un article spécifique. C'est une incohérence ${ }^{39}$. » Les différences d'un lieu à l'autre n'étaient souvent pas très importantes, mais si chacun agissait à sa convenance, si chaque province avait ses propres lois, le manque d'harmonisation et de régularisation au sein d'un même système juridique étatique était alors patent. Par exemple, pour le même crime de "vol furtif », quelqu'un pouvait être condamné à porter les fers, voire à être attaché à un poteau ou bien à un rocher, selon qu'il tombait sous le coup de lois spéciales à la province du Hunan, du Hubei, du Fujian, du Guangdong, Yunnan, Shandong, Anhui, Zhili, Sichuan, Shaanxi ou du Gansu : chacune avait des lois spéciales en ce sens. Selon un

38. Voir l'article de Frédéric Constant dans ce volume.

39. Xue 1970: 717 (Art. 273-16). 
commentaire critique de Xue Yunsheng : «Bien que chaque province présente des circonstances particulières, si l'on veut punir les bandits avec une égale sévérité, il ne faut pas qu'il y ait une loi pour chaque province, ce qui ne peut qu'entraîner des contradictions. Il faut revoir et comparer tous ces article en vue de les uniformiser ${ }^{40}$. » En outre, les lois spéciales n'existaient qu'en référence aux lois générales, mais comme les canaux de transmission des mémoires proposant des lois nouvelles n'étaient pas les mêmes, certaines lois générales furent actualisées, alors que les lois spéciales ne connaissaient pas les ajustements correspondants.

Afin de remédier à ces lacunes, les gouvernants s'efforcèrent de prendre des mesures rectificatives. La principale d'entre elles fut d'entreprendre une révision générale des anciens articles afin de mettre en œuvre une uniformisation de la législation. De nombreuses lois spéciales à caractère régional entrèrent dans le processus de grande révision des articles additionnels du code, consistant en « ajout, suppression, amendement, fusion ». Lorsque les conditions étaient suffisamment mûres, certaines lois spéciales devinrent des lois générales à tout l'empire. Ainsi, un article additionnel annexé en 1834 à l'article «Franchir clandestinement une frontière et transgresser l'interdiction de se rendre en mer » (Art. 225) dispose :

À Taiwan, un malfaiteur qui purifie du salpêtre ou du soufre, qu'il en fasse ou non commerce, pour une quantité allant jusqu'à dix livres : cent coups de bâton, impression du tatouage légal et renvoi forcé sur le continent. Pour dix livres et plus : soixante coups de bâton et un an de servitude. Pour chaque dix livres supplémentaires, augmenter d'un degré. Pour cent livres, ou leur transformation en poudre à canon, jusqu'à dix livres : exil militaire sur une frontière proche. Pour plus de trois cents livres, ou plus de dix livres de poudre à canon, conformément à l'article relatif au fait de couler privément des pièces d'artillerie de grande ou petite taille appelées communément « Habit rouge » : décapitation ; les épouse et enfants sont incriminés solidairement, le patrimoine est confisqué au profit de l'administration. Si le salpêtre ou le soufre ont été vendus à des aborigènes ou transportés clandestinement par la mer, condamner comme pour les brigands en bande. Les chefs de la milice, les voisins et garants, porteurs ou patrons de barque qui connaissent les faits sans les dénoncer sont incriminés solidairement.

Cet article avait comme arrière-plan la région productrice de soufre située autour du massif volcanique de Tatun, au nord de Taiwan, et édictait des règles spécifiques à cet endroit. Dans d'autres régions, les peines étaient plus légères,

40. Xue $1970: 670$. 
limitées à l'exil militaire sur une frontière proche pour fabrication de poudre à canon à hauteur de cent livres et plus. Les personnels administratifs impliqués subissaient également des peines plus légères : «Lorsqu'un voisin ou un garant connaît les faits et ne les dénonce pas, la peine est de cent coups de bâton. S'il ne connaît pas les faits, la peine est de quatre-vingts coups de bâton. Les porteurs ou patrons de barque qui connaissent les faits sans les dénoncer sont passibles de la même peine que le criminel diminuée de deux degrés ${ }^{41}$. $\gg$ Les progrès des Taiping et l'affaiblissement du pouvoir amenèrent à réviser de vieux articles à partir de 1861. D'où l'insertion dans le code d'un nouvel article additionnel ainsi conçu : "Lorsque sur le continent, dans les régions productrices de soufre, des malfaiteurs se livrent à la purification ou à l'extraction, qu'il y ait eu ou non vente, prononcer la sentence conformément à l'article édicté pour l'île de Taiwan. » La loi spéciale était ainsi étendue en règle générale, et mis à part « le retour forcé sur le continent » prévu pour les insulaires taiwanais, le contenu était le même, moyennant de menues transformations : "Le salpêtre ou le soufre vendus à des aborigènes ou transportés clandestinement par la mer » devint « le salpêtre et le soufre fournis à des brigands », et la phrase : « les chefs de la milice, les voisins et garants, porteurs ou patrons de barque qui connaissent les faits sans les dénoncer sont condamnés solidairement » fut remplacée par celle-ci : " laisser faire en connaissance de cause, recéler et cacher au lieu de dénoncer est passible de la même peine que celle des criminels, mais si c'est la peine capitale, diminuer d'un degré ${ }^{42} »$.

\section{Conclusion}

Pour s'en tenir à l'essentiel, les défauts des lois spéciales à caractère régional provenaient d'une mauvaise répartition des pouvoirs entre les administrations centrales et locales. À peine un article était-il établi qu'un nouveau surgissait, en particulier lorsque, les troubles intérieurs succédant aux périls extérieurs, l'administration centrale ne révisa plus les lois de façon systématique : alors, des contradictions surgirent incessamment entre lois générales et lois spéciales, ou au sein des lois spéciales elles-mêmes, sans que les problèmes de fond pussent être réglés.

Sous les Qing, les lois spéciales à caractère régional n'existaient qu'au travers des articles additionnels édictés par l'administration centrale, de sorte qu'aucun pouvoir législatif local ne fut jamais reconnu. Ce n'est qu'à

41. Xue $1970: 520$ (Art. 225-39).

42. Xue $1970: 478$ (Art. 214-05). 
la toute fin des Qing, lors des travaux préparatoires à l'établissement de la constitution, que l'autonomie locale devint une question importante. Les Règlements relatifs aux bureaux consultatifs de chaque province (Gesheng ziyiju zhangcheng) promulgués à la fin de l'ère Guangxu déterminaient pour la première fois les prérogatives législatives des provinces. En 1913, le sénat provisoire promulgua une loi temporaire autorisant les assemblées provinciales à édicter des règlementations spéciales pour leur province ${ }^{43}$. Dès les débuts de la République, Sun Yat-sen avait prôné l'équilibre des pouvoirs aux niveau local et central, ainsi que l'autonomie locale, tout en préconisant que chaque district puisse avoir ses propres lois décidées par une assemblée de députés élus. Dans sa Proclamation de prise de fonction de la présidence à titre provisoire, il envisageait l'établissement de «constitutions provinciales » par chaque province. Ce sont ici les prémisses d'une réflexion de fond sur la législation locale ${ }^{44}$. Les articles 24 à 28 du chapitre 5 (« Souveraineté nationale ») de la Constitution de la République de Chine de 1923, garantissant aux provinces un pouvoir législatif autonome dès lors que celui-ci n'entrait pas en conflit avec le droit national, furent la première dévolution de pouvoir législatif au niveau local par une constitution chinoise. Les bouleversements affectant le système politique entravèrent l'application réelle de ces dispositions, mais le fait que des pouvoirs législatifs devaient s'exercer au niveau local devint l'opinion commune, tandis que les lois spéciales à caractère régional promulguées par l'administration centrale voyaient leur rôle considérablement réduit. Ceci représentait une rupture radicale avec l'esprit de la législation des Qing.

Bien évidemment, la recherche d'une distribution plus rationnelle du pouvoir de légiférer en général, et plus particulièrement dans le domaine pénal, entre administration centrale et pouvoir local, ceci dans le but d'atteindre une plus grande efficacité et une meilleure justice dans la répression des crimes aussi bien qu'un meilleur règlement des litiges civils - tout cela reste d'une actualité brûlante.

43. Xie 1948 : 808 .

44. Pan $1992: 321-322$. 
Les lois spéciales à caractère régional dans le code des Qing

\section{BIBLIOGRAPHIE}

\section{Sources primaires}

NDT. Les références bibliographiques des ouvrages antiques (Hanfeizi 韓非子, Hanshu 漢書, Guanzi 管子, Lunyu 論語, Mozi 墨子, Shangshu 尚書, Zhouli 周禮) ne sont pas indiquées dans l'article original de Wang Zhiqiang.

Dongguan xian zhi 東莞縣志 (1921).

HuANG, Zongxi 黃宗羲 (1981). Ming yi daifang lu 明夷待訪錄. Pékin : Zhonghua shuju.

FANG, Xuanling 房玄龄 (1974). Jinshu 晉書. Pékin : Zhonghua shuju.

LAN, Dingyuan 監鼎元 (1827). 《Taiwan baojia ze chengxiang changshu 台灣保甲 責成鄉長書. 》 In Huangchao jingshi wenbian 皇朝經世文編, compilé par HE Changling 賀長齡 et WEI Yuan 魏源, juan 84.

Mingdai Tiaoli 明代條例 (1994). In YANG Yifan 楊一凡 (éd.), Zhongguo zhenxi falü dianji jicheng 中國珍稀法律典籍集成, 2-2. Pékin: Kexue chubanshe.

MONTESQUIEU (1834). De l'esprit des lois. Paris : Lebigre frères.

WANG, Fengsheng 王鳳生 (1997 [1826]), Songzhou congzheng lu 宋州從政錄. In LIU Junwen 劉俊文 et al. (éd.), Guanzhen shu jicheng 官箴書集成, vol. 5. Hefei : Huangshan shushe.

WU, Hong 吳宏 (1999). Zhishang jinglun 紙上經綸. In GUO Chengwei 郭成偉 et TIAN Tao 田濤, Ming Qing gongdu miben 明清公牘秘本. Pékin : Zhongguo zhengfa daxue chubanshe.

XuE, Yunsheng 薛允升 (1970 [1905]). Duli cunyi 讀例存疑. Réédition de Huang Jingjia 黃靜嘉. Taipei : Chengwen chubanshe.

XuE, Yunsheng 薛允升 (2000 [1922]). Tang Ming lü hebian 唐明律合編. Réédition de HUAI Xiaofeng 懷效鋒. Beijing, Falü chubanshe.

Zhongguo renmin daxue, zhongguo diyi lishi dang'anguan (éd.) (1991). Qingdai nongmin zhanzheng shi ziliao xuanbian 清代農民戰爭史資料選編. Pékin : Zhongguo renmin daxue chubanshe.

\section{Sources secondaires}

GuO, Dongxu 郭東旭 (1997). Songdai fazhi yanjiu 宋代法制研究. Baoding: Hebei daxue chubanshe.

PAN, Nianzhi 潘念之 et al. (1992). Zhongguo jindai falü sixiang shi 中國近代法律思 想. Shanghai : Shanghai shehui kexue chubanshe.

SHIGA, Shūzō 滋贺秀三 (1986). «Shindai sosho seido ni okeru minjitekihogen no gaikatsuteki 清代訴訟制度における民事的法源の概括的検討 ».Tōyo shi $k e n k y \bar{u}$ 東洋史研究, 40-1: 74-102.

TERADA, Hiroaki 寺田浩明 (1993). «Shindai no shorei 清代省例 》. In Chūgoku hōseishi : kihon shiryō no kenkyū 中国法制史 : 基本資料の研究, édité par SHIGA Shūzō. Tokyo, Tōkyō Daigaku Shuppankai : 657-714. 


\section{Wang Zhiqiang}

XIE, Zhenmin 謝振民 (1948). Zhonghua minguo lifashi 中華民國立法史立法史. Shanghai : Zhengzhong shuju.

ZHOU, Zhenhe 周振鹤 (1998). Zhonghua wenhua tongzhi 中華文化通志. Shanghai :

Shanghai Renmin chubanshe.

\section{GLOSSAIRE}

Chi ling ge shi 敕令格式

daozei zhongfa 盗賊重法

fa zi jun chu 法自君出

fanjian 犯茹

Feng Guangyu 馮光裕

Fengyang 鳳陽

gaoshi 告示

Gesheng ziyiju zhangcheng 各省諮議局章程

Guide fu 歸德府

Guluzi 㖪嚕子

jingyue 禁約

Lan Dingyuan 藍鼎元

lü 律

Ming yi daifang lu 明夷待訪錄

nei zhong wai qing 內重外輕

shengli 省例

teshu zhuti 特殊主體

tiaoli 條例

tu zhengce 土政策

Wang Fengsheng 王鳳生

wenxing tiaoli 問刑條例

Xingfa zhi 刑法志

Xue Yunsheng 薛允升

yiban zhuti一般主體

Yingzhou 穎州

Zha ge zhouxian chaban baojia xi 札各州縣查辦保甲檄

Zhi Zhe chenggui 治浙成規

Zhongfadi 重法地

zili cisong 自理詞訟

ziran ren 自然人

zuo'er 佐貮 